\title{
Atom Predicates and Set Predicates: Towards a General Theory of Plural Quantification
}

\author{
Yoad Winter \\ Utrecht Institute of Linguistics OTS
}

\section{Introduction}

The interpretation of sentences with plural determiners and collective predicates is a highly complex problem that has not been sufficiently investigated, despite many advances in theories of plurality and quantification. This paper proposes a new typology of predicates that serves as a basis for a general treatment of singular and plural noun phrases within generalized quantifier theory.

Section 2 proposes that instead of the traditional distinction between "distributive", "collective" and "mixed" predicates, a binary classification of predicates should be used according to their behaviour with plural determiners like all and exactly five. Predicates that allow collectivity with such determiners are referred to as set predicates. These are predicates like meet, gather, surround the castle or praise each other, which are traditionally classified as collective/mixed. However, following Dowty (1987) it is shown that some intuitively "collective" predicates like be numerous, be a good team or vote do not show collectivity when combined with plural quantificational noun phrases. Together with "distributive" predicates, these are classified as atom predicates. It is proposed that the difference between atom predicates and set predicates lies in their type. Atom predicates range over singularities - members of an arbitrary domain of individuals $E$. Set predicates range over pluralities - non-empty subsets of $E$. This does not mean that atom predicates cannot show collectivity effects, but only that these effects are restricted to "set-denoting" noun phrases like the students or Mary and Sue. Following previous works it is proposed that collectivity with set denoting NPs may appear due to a mapping from sets to "impure" atoms in $E$ : singularities of the same sort as the denotation of NPs like the committee or the team.

Section 3 substantiates the quantificational mechanism that accounts for the meaning of sentences like all the students met, where a plural determiner appears with a set predicate. Determiners standardly range over atoms. Plural nouns, unlike singular nouns, range over sets. This type mismatch between the determiner and the plural noun triggers a type shifting principle that fits the determiner to quantification over sets. The relations between this process and the conservativity of quantification in natural language are briefly examined.

\section{Atom Predicates and Set Predicates}

Dowty (1987) shows a systematic difference between the behaviour of predicates like meet or gather and predicates like be a good team or be numerous when combined with noun phrases like all (the) students. While the first predicates give rise 
to a collective interpretation of the sentences in (1a), sentences with the latter predicates as in (1b) are highly bizarre. To the extent that they are interpretable, they are as strange as sentences like every student is numerous/a good team.

a. All the students met/gathered in the hall.

b. *All the students are numerous/a good team.

Dowty's paper concentrates on the semantics of all, but the distinction it makes between predicates is more general and applies to their behaviour with many other plural "quantificational" noun phrases. Consider the examples in (2), which show that the case of all is only one instance of a more general paradigm. ${ }^{1}$

(2) $\left\{\begin{array}{l}\text { exactly four } \\ \text { between four and ten } \\ \text { more/less than eleven } \\ \text { at least/most twelve } \\ \text { few/many } \\ \text { no } \\ \text { ?most/most of the }\end{array}\right\}$ students $\left\{\begin{array}{l}\text { met/gathered in the hall } \\ * \text { are numerous/a good team }\end{array}\right\}$

Importantly, even those predicates like be a good team, which refuse to participate in such quantificational structures, do appear felicitously with the NPs in (3).

(3) a. The students/some students I know/five students I know/Mary and John/ some student and some teacher I know/the student and the teacher are a good team.

b. Both the students and the teachers/either the students or the teachers/ neither the students nor the teachers are a good team.

Noun phrases as in (3) are often used to classify predicates like be a good team, meet or lift a piano as "non-distributive" or "collective". This is demonstrated by the lack of equivalence in (4).

(4) The students are a good team/met/lifted a piano.

$\Leftrightarrow$ Every student $*$ is a good team $/ *$ met/lifted a piano.

However, Dowty points out that the same observation applies to many intuitively "distributive" predicates. He exemplifies the problem using the sentence in (5) and the discourse in (6).

(5) At the end of the press conference, the reporters asked the president questions.

a. What was that noise?

b. Oh, I'm sure it was only the children getting up to watch cartoons. Go back to sleep.

Sentence (5) does not assert that all the reporters asked questions. Similarly, sentence (6b) does not entail that all the children are getting up. Dowty concludes:

I argue that even with 'true' distributive predicates, the question of how many members of the group referent of a definite NP must have the distributive property is in part lexically determined and in part determined by the context, and only rarely is every member required to have these properties. 
Thus, the behaviour with noun phrases as in (3) is not too useful for making a robust distributive/collective distinction between predicates. Instead of this vague typology I propose a new criterion for classifying predicates. The criterion is based on comparing the behaviour of predicates with singular quantificational noun phrases like every students to their behaviour with plural quantificational noun phrases like all (the) students.

The every/all criterion: An English predicate is called an atom predicate if and only if the sentence obtained by combining it to an every noun phrase is equivalent to the sentence we get when it is combined with an all noun phrase. Predicates that lead to non-equivalent sentences are referred to as set predicates.

The reason behind this terminology will become clear in a while. The every/all criterion is a convenient test in languages like English that clearly distinguish between universal determiners in the singular (every, each) and in the plural (all). Other languages may need slightly more sophisticated tests for the classification, according to contrasts as in (2). In general, set predicates are those predicates that show collectivity with plural determiners; atom predicates do not.

Distributive predicates like sleep, smile, and get up are clearly atom predicates, as the equivalences in (7a) show. Importantly, also "collective" predicates like be a good team and be numerous qualify as atom predicates. This is since the kind of oddness that one experiences upon hearing sentences as in (7b) is clearly the same: both with every and with all the sentence attributes the property of being a good team to each and every singular individual quantified over. For all purposes, these unacceptable sentences are equivalent. Their unacceptability reasonably follows from selectional properties of the predicate: asserting that this student is a good team is not stranger (in fact, much more reasonable) than stating that this piece of paper slept. Both unacceptabilities are not facts about truth-conditions and they are therefore insignificant for making modeltheoretic distinctions between predicates like be a good team and sleep.

a. Every student slept/got up $\Leftrightarrow$ All the student slept/got up

b. *Every student is a good team/numerous $\Leftrightarrow *$ All the students are a good team/numerous

"Mixed" atom predicates do not give rise to unacceptabilities as in (7b). For instance, as Dowty points out, the predicate vote leads to a collective effect in sentence $(8 \mathrm{a})$, where the vote need not be unanimous. That is, sentence $(8 \mathrm{a})$ is not equivalent to $(8 \mathrm{~b})$. On the other hand, sentence (8c) is equivalent to (8b), which characterizes vote as an atom predicate. A similar effect is exemplified in (9) with the verb weigh: sentence (9c), like (9b) but unlike (9a), must be interpreted as talking about heavy potatoes rather than about the weight of the whole basket.

(8) a. The students voted to accept the proposal.

b. Every student voted to accept the proposal.

c. All the students voted to accept the proposal. 
(9) a. The potatoes in this basket weigh $1 \mathrm{~kg}$.

b. Every potato in this basket weighs $1 \mathrm{~kg}$.

c. All the potatoes in this basket weigh $1 \mathrm{~kg}$.

Set predicates include collective predicates like meet, gather and be similar, as well as reciprocated predicates like admire each other. This is demonstrated by the lack of equivalence in (10).

(10) *Every student met/gathered/is similar/admired each other

$\Leftrightarrow$ All the students met/gathered/are similar/admired each other

With many "mixed" predicates like lift a piano there is probably some variation among speakers. For instance, Dowty (1987:104) recognizes a "collective interpretation" of sentence (11a), which means he reads it as non-equivalent to (11b). Thus, in Dowty's dialect the predicate perform Hamlet is a set predicate. However, Dowty also mentions that some people find collective interpretations in (11a) more natural if the word together is added. This seems to be correct also for speakers like Dowty who do not take (11a) and (11b) to be equivalent. Other speakers, however, are stricter and consider (11a) and (11b) to be totally equivalent. For these speakers, only when together is added can the sentences become non-equivalent. Using the present terminology, in this dialect the predicate perform Hamlet is an atom predicate but together modifies it into a set predicate. A similar point holds for many other "mixed" predicates like lift a piano or write a book.

a. All the students in my class performed Hamlet.

b. Every student in my class performed Hamlet.

Moving to the nominal domain, it is important to note that the every/all criterion classifies predicates as atom or set independently of their morphological number. Thus, the plural form of a nominal gets the same classification as its singular form. For instance, both the singular noun student and its plural form students are classified as atom predicates due to the equivalence in (12). Nouns like colleagues, brothers, friends and so on qualify as set predicates according to non-equivalences as in (13a). Another way to obtain a (complex) set nominal is to modify an atom nominal by a set predicate as illustrated in (13b-c).

(12) Every woman is a student

$\Leftrightarrow$ All the women are students

a. Every woman is a friend $\Leftrightarrow$ All the women are friends

b. Every woman is a similar student $\Leftrightarrow$ All the women are similar students

c. *Every woman is a student who met yesterday at school $\Leftrightarrow$ All the women are students who met yesterday at school

The following list gives a summary of atom predicates and set predicates according to the every/all criterion.

(14) Atom predicates: 
a. sleep, smile, get up

b. be a good team, be numerous, form a triangle, elect Clinton, constitute a majority, outnumber (both arguments)

c. vote to accept the proposal, weigh $1 \mathrm{~kg}$

d. (in some dialects:) perform Hamlet, lift a piano, write a book

e. student(s), child(ren), shop(s)

(15) Set predicates:

a. meet, gather, disperse

b. be similar, be alike

c. like each other, look at one another

d. perform Hamlet together, lift a piano together, write a book together

e. (in some dialects:) perform Hamlet, lift a piano, write a book

f. colleague(s), brother(s), similar student(s), student(s) who met

Thus, an atom predicate can be distributive (e.g. sleep), collective (e.g. be a good team) or mixed (e.g. vote). All set predicates are collective (or mixed, in some dialects), but not vice versa. Logically, all distributive predicates are atom predicates.

It should be stressed again that the atom/set distinction, although made using the every/all criterion, applies to all plural determiners in (2), as witnessed by the contrast between the predicates in these examples. Furthermore, with "mixed" predicates we can observe an atom/set contrast that does not involve unacceptability effects as in (2). For instance, sentence (16) states that the total number of individual students who voted to accept the proposal is ten. The sentence does not leave open the possibility that the number of students who made an individual affirmative vote was more than ten or less than ten. Roughly speaking, with atom predicates the quantification fully distributes to atoms. By contrast, sentence (17) contains the predicate drink a whole glass of beer, which in the "Dowty dialect" is a set predicate. In this dialect the sentence can be true in case ten students drank one whole glass of beer together, but no student drank a whole glass of beer on her own. Thus, plural quantification with set predicates takes into account also group actions.

(16) Exactly ten students voted to accept the proposal.

(17) Exactly ten students drank a whole glass of beer.

The label atom or set for a predicate is used descriptively to refer to its behaviour under the every/all criterion, and with plural quantifiers in general. The terms are chosen according to the basic idea that will be used to account for the differences between the two classes.

The atom/set hypothesis: Atom predicates range over singular individuals. Set predicates range over sets of singular individuals.

In a type-theoretical analysis of plurals (e.g. Bennett (1974)), singularities are objects of type $e$ and plural individuals are of type $e t$. Let us adopt this assump- 
tion for technical convenience. In Bennett's typing the atom/set hypothesis means that the basic denotation of atom predicates is et whereas set predicates basically denote ett predicates. There are now two standard assumptions that will be used to account for the differences between the two classes of predicates when combined with various NPs. The first assumption is that all determiners basically range over atoms. That is, expressions like every, all, exactly five, no, between five and ten etc. standardly denote relations between sets of atoms: they have the type $(e t)(e t t)$ as in classical generalized quantifier theory. Note that in this standard setting there is no denotational difference between every and all or between singular no and plural no. I argue that there is no need to impose such denotational differences in order to account for the meaning differences between these items.

The second standard assumption is that "referential" plurals like the students or Mary and John denote pluralities - they are of type et (sets of atoms). There are two major simplifications that are made in this assumption. First, I assume that these NPs refer to sets, while in fact coordinations like neither the students nor the teachers as in (3b) show that we need to have complex boolean operations on such plural individuals for which sets are insufficient. This is naturally done in the Montagovian way, by dealing with principal ultrafilters over plural individuals, of type $((e t) t) t$, instead of just "referential" sets. A second complication is that indefinite noun phrases like three students I know are not assumed to be "referential" (contra Fodor and Sag (1982) a.o.), but as in Reinhart (1997) and Winter (1997) they denote sets (or their principal ultrafilters) which are picked by choice functions. Ignoring these irrelevant complications, we end up with four possibilities to combine atom/set predicates with quantificational/"referential" NPs. The first two possibilities are straightforward:

1. When combining an atom predicate like sleep or be a good team with a quantificational noun phrase like every student, all students or exactly five students we simply apply a standard ett quantifier to an et predicate. This standardly captures distributivity effects as in (7).

2. When a set predicate like meet has a "referential" argument like the students, we get a collective effect due to the application of an ett predicate to an et plural individual argument.

Sentence (18) is a more complex case where an atom predicate meets a "referential" set argument.

(18) The students are a good team.

We start from the natural assumption that the singular predicative indefinite $a$ good team denotes a predicate over atoms. A "team atom" in the extension of such a predicate is sometimes called "impure" because it is (at least pragmatically) related to a set of other atoms: the set of the team members. To combine the denotation of the atom predicate be a good team with the set denotation of the noun phrase the students we have to resolve the type mismatch between these objects. Such a resolution operation is something that all present theories of plurality assume, with 
differences in implementation. Following Landman (1996), let us assume that NPs that denote a set can be freely mapped to a corresponding atom. Specifically, the set denoted by the noun phrase the students can be mapped to an "impure" atom it constitutes. If this atom turns out to be in the extension of the atom predicate be a good team then sentence (18) is true. For more on such processes, see Barker (1992), Landman (1989) and Schwarzschild (1996:ch.9). I assume that this strategy is responsible also for non-distributivity effects as in (3), (5), (6), (8a) and (9a).

The fourth case is our main concern. This is the case where a set predicate like meet appears with a quantificational noun phrase. In this situation what happens depends on the morphological number of the noun phrase. For instance, contrast the plural noun phrases in (20) with the singular noun phrases in (19).

a. *Every student is meeting.

b. *More than one student is meeting.

c. *No student is meeting.

a. All the students are meeting.

b. At least two students are meeting.

c. No students are meeting.

The emerging generalization is obvious: morphological plurality is a necessary condition for a noun phrase to be "collective". 2 The next section proposes a mechanism that captures this generalization and accounts for the semantics of collective quantification as in (20).

\section{Quantification over sets}

In Bennett's typing the "semantic number" of a predicate is modeled by its type. A predicate ranging over atoms (singularities) is of type et - a function that characterizes a subset of the domain $E$ of entities. A predicate over collections of atoms is of type ett - a function that characterizes a subset of the power-set $\wp(E)$ of the domain of entities. In the proposed analysis, two factors determine the type (the "semantic number") of a natural language predicate (noun, verb or adjective). The first factor is morphological number (e.g. student vs. student $\underline{s}$ ). The second factor is the atom/set distinction as determined by the every/all equivalence criterion. We adopt the following principles:

1. Morphologically singular predicates in natural language unambiguously denote $e t$ predicates.

2. Morphologically plural predicates in natural language are ambiguous between type $e t$ and type ett with systematic lexical operations connecting the two denotations:

(a) Plural atom predicates are basically of type $\underline{\text { et }}$ and they get their ett denotation by a distributivity operator pdist. 
(b) Plural set predicates are basically of type ett and they get their et denotation by a singularity operator $s g$.

The pdist operator is the familiar distributivity operator on predicates mapping a set to the set of its non-empty subsets. The singularity operator $s g$ maps a set of sets $\mathcal{A}$ to the set of entities that constitute singletons in $\mathcal{A}$. Formally:

$$
\begin{array}{lll}
\operatorname{pdist}_{(e t)(e t t)} & \stackrel{\text { def }}{=} & \lambda A \cdot \lambda B \cdot \emptyset \neq B \subseteq A \\
s g_{(e t t)(e t)} & \stackrel{\text { def }}{=} & \lambda \mathcal{A} \cdot \lambda x \cdot \mathcal{A}(\{x\})
\end{array}
$$

These two operators can be easily generalized to predicates of arbitrary arity. I spare the general definitions and sloppily denote by pdist $^{m}$ and $s g^{m}$ the operators that manipulate the $m$-th argument of an $n$-ary predicate, where $n$ is obvious from the context. Thus, for one place predicates we have pdist ${ }^{1}=$ pdist and $s g^{1}=s g$.

The effects of the parameters singular/plural and atom/set on the type and the denotation of predicates are summarized in table 1. The notation $A$ stands for a denotation of type $e t$, while calligraphic $\mathcal{A}$ stands for an object of type ett.

\begin{tabular}{|l|l|l|}
\hline & singular & plural \\
\hline atom & $A_{e t}$ & $A, p \operatorname{dist}(A)$ \\
set & $s g(\mathcal{A})$ & $\mathcal{A}_{\text {ett }}, \operatorname{sg}(\mathcal{A})$ \\
\hline
\end{tabular}

Table 1: types and the properties singular/plural and atom/set

To give an example, the noun student(s) is an atom predicate. The denotation of its singular form is unambiguously the et predicate student'. The plural form students is ambiguous between student ${ }^{\prime}$ and students ${ }^{\prime}=p \operatorname{dist}\left(\right.$ student $\left.^{\prime}\right)$, the set of the non-empty subsets of student'. A similar analysis is adopted for the singular atom predicate is numerous and its plural form are numerous. By contrast, a set predicate like meet basically denotes the ett object meet'. In singular (as in is meeting) we take only $s g$ (meet'). In plural (as in are meeting) the verb exposes its set nature: it is ambiguous between meet $^{\prime}$ and $s g$ (meet ${ }^{\prime}$ ).

A distributivity procedure like the pdist operator is widely accepted as a necessary mechanism to account for the quantificational effects with "referential" plurals. For instance, sentence (22) can be true in case there is a piano that the girls are lifting. This is captured by the "collective" reading in (22a), with no application of pdist. However, the sentence can also be true in case every girl is lifting a different piano. This is captured by application of pdist to the denotation of the complex predicate lift a piano as in (22b). ${ }^{3}$ The distributive interpretation is more prominent, for obvious reasons, in sentences like the girls are wearing a dress.

(22) The girls are lifting a piano.

a. $\left(\lambda x . \exists y\left[\operatorname{piano}^{\prime}(y) \wedge \operatorname{lift}^{\prime}(y)(x)\right]\right)(G)$ $\Leftrightarrow \exists y\left[\operatorname{piano}^{\prime}(y) \wedge \operatorname{lift}^{\prime}(y)(G)\right]$

b. $p \operatorname{dist}\left(\left(\lambda x . \exists y\left[\right.\right.\right.$ piano' $^{\prime}(y) \wedge s g^{2}\left(\right.$ lift $\left.\left.\left.\left.^{\prime}\right)(y)(x)\right]\right)\right)(G)$ $\Leftrightarrow G \subseteq\left(\lambda x . \exists y\left[\operatorname{piano}^{\prime}(y) \wedge s g^{2}\left(\right.\right.\right.$ lift $\left.\left.\left.^{\prime}\right)(y)(x)\right]\right)$ 


$$
\begin{aligned}
& \Leftrightarrow \forall x \in G \quad \exists y\left[\text { piano }^{\prime}(y) \wedge s g^{2}\left(\text { lift }^{\prime}\right)(y)(x)\right] \\
& \Leftrightarrow \forall x \in G \exists y\left[\text { piano }^{\prime}(x) \wedge \operatorname{lift}^{\prime}(y)(\{x\})\right]
\end{aligned}
$$

Following Landman (1989), the distributivity operator is used for all natural language predicates: verbs, nouns and adjectives. With verbs it accounts for quantificational distributivity as in (22) and with nouns it accounts for "cumulative reference" (if $x$ is a student and $y$ is a student, then $x$ and $y$ are students). ${ }^{4}$

The singularity operator $s g$ is not very central in my proposal and its only need is to make the relation between the denotations of lexical plural set predicates transparent, as the "basic" denotation of such predicates is a set of pluralities. This operator is used only as a lexical strategy for deriving ambiguity of plural set predicates, and as far as I know it is not needed for cases of complex predicates, unlike the pdist operator (cf. (22)).

After defining the atom/set distinction and its relations with morphological number and the type of plural predicates we may turn to the central question of this section: how do quantificational NPs combine with set predicates? Consider for example sentence (23) below.

(23) Exactly five students met.

The plural noun students and the verb meet can denote ett predicates: students ${ }^{\prime}=$ pdist(student') and meet' respectively. The determiner exactly five standardly denotes the relation exactly $5^{\prime}$ that holds between $\underline{e t}$ predicates whose intersection is of cardinality five. The resolution of this type mismatch is the point where quantification over sets comes into play. Unlike previous works, notably Scha (1981) and van der Does (1993), which assume a multitude of operators for quantification over sets, I propose a uniform process that adjusts the $(e t)(e t t)$ determiner to its ett arguments. Conceptually, the result of this process is that both arguments are lowered to et predicates so ordinary quantification can apply. This is obtained in two steps, which are illustrated below for sentence (23).

Step I (intersection): The verb denotation meet' is modified by intersecting it with the noun denotation students'. We get the set meet' $\cap$ students', the set of all non-empty sets of students that met. As will be explained below, this operation is motivated by the conservativity property of quantification in natural language.

Step II (union): The sets of sets that serve as arguments of the determiner are both unioned. Thus, the set of sets students' is lowered to the set Ustudents', which is just the singular noun denotation student'. The intersection set of sets meet $^{\prime} \cap$ students' is lowered to $\cup\left(\right.$ meet $^{\prime} \cap$ students $\left.{ }^{\prime}\right)$ : the set of students who participated in a set of students that met. This notion of participation is taken to be central to the semantics of plurals.

The resulting reading is calculated in (24) and paraphrased in (25).

(24) exactly_5' $5^{\prime}\left(\cup\right.$ students $\left.{ }^{\prime}\right)\left(\cup\left(\right.\right.$ meet $^{\prime} \cap$ students $\left.\left.^{\prime}\right)\right)$

$\Leftrightarrow$ exactly_5 $5^{\prime}$ student $\left.{ }^{\prime}\right)\left(\cup\left\{A \subseteq\right.\right.$ student $\left.\left.^{\prime}: A \neq \emptyset \wedge \operatorname{meet}^{\prime}(A)\right\}\right)$

$\Leftrightarrow$ exactly_5 $5^{\prime}$ student $\left.^{\prime}\right)\left(\left\{x_{e}: \exists A \subseteq\right.\right.$ student $^{\prime}\left[x \in A \wedge\right.$ meet $\left.\left.\left.^{\prime}(A)\right]\right\}\right)$ 


$$
\Leftrightarrow \mid\left\{x \in \text { student }^{\prime}: \exists A \subseteq \text { student }^{\prime}\left[x \in A \wedge \text { meet }^{\prime}(A)\right]\right\} \mid=5
$$

(25) The number of students who participated in a student meeting is five.

As in the works of Scha and van der Does, we can implement the two step strategy above using a lifting operator that maps standard $(e t)(e t t)$ determiners to higher type determiners. However, unlike the Scha and van der Does proposals, also the nominal argument of the lifted determiner is an ett predicate and hence the determiner is of the "symmetric" type $(e t t)((e t t) t)$ : a relation between ett predicates over plural individuals. The lifting process is referred to as determiner fitting, or $d f i t$ in short. This operator is defined below in general.

(26) Determiner fitting: $\operatorname{dfit}(D) \stackrel{\text { def }}{=} \lambda \mathcal{A} \cdot \lambda \mathcal{B} \cdot D(\cup \mathcal{A})(\cup(\mathcal{A} \cap \mathcal{B}))$

Before moving to empirical matters it is important to clarify the intuitive background behind the definition of the dfit strategy and its relations with generalized quantifier theory. Under the slogans "conservativity as intersection" and "participation as union" below I explicate the process a bit more.

\subsection{Conservativity as intersection}

Step 1 of the dfit process, where the verb denotation is intersected with the noun denotation, is closely related to the conservativity property of determiners in generalized quantifier theory. There are various ways to conceive of this familiar notion. A linguistically attractive one is to view it as an empirical generalization on quantification in natural language. Consider a sentence of the form Det-N-VP. Conservativity means that any sentence of this form is semantically equivalent to a sentence of the same form where the verb phrase is replaced by an expression that has the effect of intersecting the original verb phrase denotation with the noun denotation. One option to get such an effect is conjunction, as the equivalence in (27) illustrates. Another option is relative clause formation, as exemplified by the equivalence in (28). Yet another intersective strategy is using "intersective" adjectives like the adjective pregnant in (29). It is commonly agreed that equivalence patterns similar to (27)-(29) apply with all syntactic determiners in natural language.

(27) Every student is a woman $\Leftrightarrow$ Every student is a student and a woman

(28) Every student arrived $\Leftrightarrow$ Every student is a student who arrived

(29) Every student is pergnant $\Leftrightarrow$ Every student is a pregnant student

The common way to account for conservativity equivalences is by appealing to a model-theoretic property of natural language determiners. This is the familiar definition given below.

(30) A determiner denotation $D$ is conservative iff for every $A, B \subseteq E$ : $D(A)(B) \Leftrightarrow D(A)(A \cap B)$.

Under this perspective, conservativity appears in natural language because, so it happens, all the denotations of natural language determiners are conservative functions. As far as I know there is no theory that clearly accounts for why this happens to be the case. 
There is an alternative, less standard way to approach conservativity. ${ }^{5}$ Suppose that by some means of syntax or by way of semantic composition the second argument of a determiner is always intersected with the first argument before application occurs. Thus when the denotations of the determiner, the noun and the verb phrase are $D, A$ and $B$, respectively, the meaning of the sentence is not $D(A)(B)$ as usual, but rather $D(A)(A \cap B)$. This accounts for conservativity equivalences as well. Crucially, however, this is so even if the denotation of the determiner $D$ is not a conservative function as defined in (30). For instance, the classical denotation of the determiner every is the subset relation as defined in (31a). Suppose, for sake of the mental excercise, that the denotation of every is the identity relation as defined in (31b). Given an intersective procedure as described above both possibilities lead to the correct analysis of a sentence like every student arrived as expressing a subset relation between the two relevant sets. This is shown in (32a-b) using the corresponding definition of every in (31a-b). The identity relation, however, is not a conservative determiner denotation, as can be easily verified.

$$
\begin{aligned}
& \text { a. } \operatorname{subset}(A)(B)=1 \Leftrightarrow A \subseteq B \\
& \text { b. } i d(A)(B)=1 \Leftrightarrow A=B \\
& \text { a. } \llbracket \text { every } \rrbracket=\text { subset: } \\
& \text { 【every student arrived } \rrbracket=1 \\
& \left.\Leftrightarrow \text { subset (student }{ }^{\prime} \text { ) (student }{ }^{\prime} \cap \text { arrive }^{\prime}\right)=1 \\
& \Leftrightarrow \text { student }^{\prime} \subseteq \text { student }^{\prime} \cap \text { arrive }^{\prime} \\
& \Leftrightarrow \text { student }^{\prime} \subseteq \text { arrive } \\
& \Leftrightarrow i d\left(\text { student }^{\prime}\right)\left(\text { student }^{\prime} \cap \text { arrive }^{\prime}\right)=1 \\
& \Leftrightarrow \text { student }^{\prime}=\text { student }^{\prime} \cap \text { arrive }{ }^{\prime} \\
& \Leftrightarrow \text { student }^{\prime} \subseteq \text { arrive }^{\prime}
\end{aligned}
$$

Getting back to plurals, the present $d$ fit operation is based on the latter view of conservativity. More formally, when both the noun and the verb phrase predicates are pdistributed et predicates, what we get is a classical conservativity equivalence that does not hinge on a conservative denotation of the determiner. Formally:

Proposition 1 For any determiner $D$, for all $A, B \subseteq E$ : $\operatorname{dfit}(D)(p \operatorname{dist}(A))(p \operatorname{dist}(B)) \Leftrightarrow D(A)(A \cap B)$.

Of course, since we assume that all predicates, singular and plural, can denote et predicates without being lifted by the pdist operator, we still have to assume the standard conservative denotations for determiners in the non-lifted case. However, if the "intersective" view on consrvativity as external to the determiner denotation is correct, it may turn out that the intersection step within dfit is a manifestation of a more general process in natural language.

Empirically interesting questions about conservativity with plurals emerge when we consider cases where one of the arguments of the determiner is a set predicate. Intuitively, the following pairs of sentences are equivalent. 
a. All the students are similar.

b. All the students are similar students.

a. All the students who met yesterday at the nightclub are rich.

b. All the students who met yesterday at the nightclub are rich students who met yesterday at the nightclub.

a. All the students who met yesterday at the nightclub are similar.

b. All the students who met yesterday at the nightclub are similar students who met yesterday at the nightclub.

The intersective process within the $d f i$ strategy accounts for such equivalences, which are not even expressible using the standard conservativity restriction in (30) on $(e t)(e t t)$ determiner denotations.

\subsection{Participation as union}

The intersective strategy in step 1 of the $d f i t$ operation still does not resolve the type mismatch between the determiner and its ett arguments, although as we will see below it is a prerequisite for a semantically sound resolution of the mismatch. To do that, we appeal to the notion of participation as in Link (1983:310), which is also the intuitive background for Dowty's account of contrasts as in (1). ${ }^{6}$ Link and Dowty, however, introduce participation operators as new semantic primitives. Instead, I propose to use the simple union operation on ett predicates. Thus, a singularity $x$ participates in a meeting if and only if it belongs to a set $A$ in the extension of meet. Put differently: $x \in \cup$ meet'. Of course, it would be a mistake to use only a union strategy in order to resolve the type mismatch. For instance, the meaning of sentence (36) cannot be modelled as in (37), paraphrased in (38).

(36) All politicians are similar.

(37) all $^{\prime}(\cup$ politicians') (Usimilar')

(38) Every politician belongs to a set of similar things.

To see the problem, suppose that every politician is similar to his mother, but no politician is similar to any other politician. Sentence (36) is clearly false, but (38) is true. The predictions we get once we use the intersective strategy before unioning the ett predicates are more adequate. For instance, in the situation just described the formula (39) and its paraphrase in (40) are both false.

(39) all $^{\prime}\left(\cup\right.$ politicians $\left.{ }^{\prime}\right)\left(\cup\left(\right.\right.$ politicians $^{\prime} \cap$ similar $\left.\left.^{\prime}\right)\right)$

(40) Every politician belongs to a set of one or more similar politicians.

Below I give some more examples for the application of the $d$ fit strategy and indicate how it can be improved so to treat a problem it encounters.

\subsection{Examples}

Consider the following sentence.

(41) Exactly two students in this room are similar. 
Consider now a situation $\mathbf{S}$ in which there are some people in the room, only three of whom are similar. These three people are $s_{1}, s_{2}$ and $t$, where $s_{1}$ and $s_{2}$ are students and $t$ is not a student but a teacher. Sentence (41) is intuitively true in this situation. It may be thought that the above mechanism fails to capture this fact because of the following consideration. The denotation of the set predicate be similar in the situation $\mathbf{S}$ may be thought to contain just one set: the set $\left\{s_{1}, s_{2}, t\right\}$ of the similar people in the room. We denote similar ${ }^{\prime}=\left\{\left\{s_{1}, s_{2}, t\right\}\right\}$. However, the intersection students ${ }^{\prime} \cap\left\{\left\{s_{1}, s_{2}, t\right\}\right\}$ is empty, since the set $\left\{s_{1}, s_{2}, t\right\}$ includes a non-student $t$ and hence it is not a subset of student'. Under such an analysis the $d f i t$ strategy would take sentence (41) to be false in situation $\mathbf{S}$ because the cardinality of the union set $\cup$ (students' $\left.\cap\left\{\left\{s_{1}, s_{2}, t\right\}\right\}\right)$ is zero rather than two.

The flaw in the above reasoning is obvious: the predicate similar' holds of all the sets of similar things, not only of one set. In fact, the predicate be similar is an example for a downward monotone set predicate, at least when small sets are involved. For instance, the following entailment holds.

(42) Mary, Sue and John are similar $\Rightarrow$ Mary and Sue are similar

Thus, in situation $\mathbf{S}$ there is a set of similar things that is a subset of the set of students: the set $\left\{s_{1}, s_{2}\right\}$. Consequently, the $d f i t$ strategy does capture the truth of sentence (41) in situation $\mathbf{S}$.

Many set predicates display such a "downward monotone" behaviour, which is often much vaguer than in the case of the predicate be similar. For instance, van der Does (1993) considers examples like the following.

(43) Exactly one hundred children met near Amsterdam.

The sentence can be true in a situation where there were many meetings near Amsterdam, some of them containing children as well as non-children. It is very hard to decide on an "intuitive" denotation of the predicate meet near Amsterdam under such a description of the situation, because whenever a set $A$ is in the extension of meet, also subsets of $A$ that are relatively large are reasonably in this extension, with fuzzy borderlines as for what "relatively large" means.

This vagueness is not so interesting as a theoretical question: after all, it is not denotations that we are after but entailment relations between sentences. However, such effects make it hard to test the predications of the dfit strategy with set predicates like be similar or meet. Fortunately, there are set predicates that show no sign of monotonicity. Contrast the following sentence with sentence (41).

(44) Exactly two students drank together a whole glass of beer.

In a situation like $\mathbf{S}$, where the only set that drank together a whole glass of beer is $\left\{s_{1}, s_{2}, t\right\}$, sentence (44) is clearly false. In this case there is no room for speculating that the set $\left\{s_{1}, s_{2}\right\}$ must be in the extension of the verb phrase: the teacher $t$ might have contributed to the "group achievement", in which case $s_{1}$ and $s_{2}$ did not drink together a whole glass of beer. In this case the $d$ fit strategy respects naive intuitions and treats sentence (44) as false.

One conclusion from these facts is that in order to check the predictions of a quantificational strategy over sets we better concentrate on non-monotone predi- 
cates as in (44). A second conclusion is that conservativity equivalences are crucial for establishing such a procedure: in both (41) and (44) truth depends only on the elements in the extension of the noun that satisfy the predicate: to determine the truth-value of (41) we are interested only in sets of students in the extension of the predicate be similar. Sets that include non-students are irrelevant for the truth of the sentence, as the equivalences in (33)-(35) imply. Likewise, in (44) we are interested in a set that drank a whole glass of beer only if it does not contain any non-student.

So far, we have not seen any motivation for the assumed ambiguity of plural nouns. The motivation for this ambiguity is that even with set predicates there is an option to quantify over atoms and ignore sets in the extension of the predicate. Consider the following sentence.

(45) Exactly five students drank a whole glass of beer.

a. $d f i t\left(\right.$ exactly_5 $\left.{ }^{\prime}\right)\left(\right.$ students $\left.^{\prime}\right)\left(\right.$ drink_beer $\left.^{\prime}\right)$ $=$ the number of students who participated in a set of students that drank a whole glass of beer is five

b. exactly_5' (student $\left.{ }^{\prime}\right)\left(s g\left(\right.\right.$ drink_beer $\left.\left.^{\prime}\right)\right)$

$=$ the number of students who drank a whole glass of beer on their own is five

The sentence can be true in case only one glass of beer was drunk and the drinker was a set of five students. This is captured by the $d$ fit strategy that the ett denotation of the plural noun triggers. The derived reading is formulated and paraphrased in (45a). However, sentence (45) can be true in situations as depicted in figure 1, where five students each drank a whole glass of beer and in addition there were three students who drank a whole glass of beer together. Although the total number of students participating in sets drinking a whole glass of beer is actually eight, sentence (45) may get a distributive reading that ignores this fact and is sensitive only in students who drank a whole glass of beer on their own. This is captured because sentence (45) is also assigned the reading in (45b).

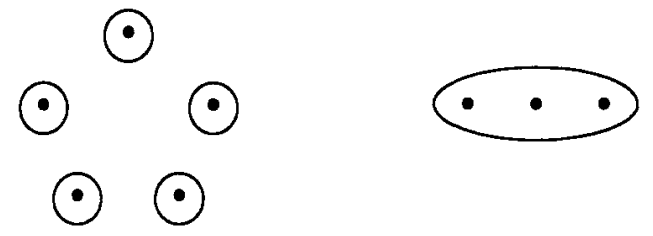

Figure 1: sets drinking beer

Another example for quantification over atoms is the analysis of simple sentences like (46), with the atom predicate sleep. Here the two strategies generate the standard "distributive" reading (46a) as well as reading (46b), which is equivalent to (46a) due to proposition 1.

(46) Exactly five students slept. 

a. exactly_ $5^{\prime}\left(\right.$ student $\left.^{\prime}\right)\left(\right.$ sleep $\left.^{\prime}\right)$
b. $\operatorname{dfit}\left(\right.$ exactly_5 $\left.^{\prime}\right)\left(\right.$ students $\left.^{\prime}\right)\left(\operatorname{pdist}\left(\right.\right.$ sleep $\left.\left.^{\prime}\right)\right)$

\subsection{The witness condition}

A problem with the $d f i$ strategy can be noticed in the analysis (39) of sentence (36) above, as paraphrased in (40). Sentence (36) requires that there is a property that is shared by all politicians. However, the analysis in (39) can be true if there are some sets of politicians, each of them in the extension of the predicate be similar, but the set of politicians itself is not in the extension of the predicate. The problem becomes more evident in examples like the following.

(47) Exactly five students drank a whole glass of beer together.

This sentence entails that there was a set of five students who together drank a whole glass of beer. To see this, consider a situation where two students drank a whole glass together and three other students drank a whole glass together as well. Intuitively, sentence (47) is not verified by this situation. However, the $d$ fit strategy only requires that the total number of students in sets of students that drank a whole glass is five. Thus, (47) is modeled as true in the given situation.

I believe that this problem is not directly related to the "static" formulation of the $d f i t$ strategy, but it is a manifestation of the context change potential of plural determiners. A sentence like (47) can be followed by a sentence like they got drunk, where they refers to a single set of five students. To capture similar anaphora effects, Szabolcsi (1997) proposes to use existential quantification over witness sets. The definition of this notion is given below.

(48) For a determiner $D$ and sets $A$ and $W \subseteq E$, we say that $W$ is a witness set of $D$ and $A$, and denote wit $(D)(A)$ iff $W \subseteq A$ and $D(A)(W)$ holds.

Szabolcsi proposes to use quantification over witnesses only for upward monotone NPs. For instance, the discourse in (49) is analyzed as in (50).

(49) More than six students passed the exam. They prepared well.

(50) $\exists W \in$ wit (more_than_6' ${ }^{\prime}$ (student') $\left[\operatorname{pdist}\left(\mathbf{p a s s}^{\prime}\right)(W) \wedge \operatorname{pdist}\left(\right.\right.$ prepare $\left.\left.^{\prime}\right)(W)\right]$

The problem in extending Szabolcsi's approach into a general strategy of existential quantification over witnesses is the interpretation of non-upward-monotone determiners like no or exactly five. For instance, analyzing (51) as in (50) would make an absurd claim requireing that a set of zero students passed the exam - the empty set is the only witness of the quantifier denoting the noun phrase no students.

(51) No students passed the exam.

To avoid this problem I propose to use quantification over witnesses as a general operation with all determiners, but unlike Szabolcsi's proposal this process is added as a condition on top of the more conventional quantificational technique of the $d f i t$ strategy. We assume the following condition.

(52) The witness condition: In a quantificational structure $D, \mathcal{A}, \mathcal{B}$ where $D$ is an $(e t)(e t t)$ determiner and $\mathcal{A}$ and $\mathcal{B}$ are ett predicates, if the intersection set 
$\mathcal{A} \cap \mathcal{B}$ is not empty then it contains a witness set of $D$ and $\cup \mathcal{A}$.

In formula, we define the relation witc between $D, \mathcal{A}$ and $\mathcal{B}$ as follows.

(53) witc $(D)(\mathcal{A})(\mathcal{B}) \stackrel{\text { def }}{\Leftrightarrow}[\mathcal{A} \cap \mathcal{B} \neq \emptyset \rightarrow \exists W \in \mathcal{A} \cap \mathcal{B}[D(\cup \mathcal{A})(W)]]$

Read: the witness condition holds between $D, \mathcal{A}$ and $\mathcal{B}$.

This additional condition guarantees that in (47) there is a witness set of the determiner exactly $\_$' ' and the predicate student', namely a set of five students, that drank together a whole glass of beer. Independently, the $d f i t$ operator respects the non-monotonicity of the determiner and guarantees that no more than five students participated in sets that drank whole glasses of beer. The double strategy leads to an analysis of (47) that is paraphrased as follows.

(54) The number of students who participated in sets of students that drank together a whole glass of beer is five (dfit) AND if there are any sets of students who drank together a whole glass of beer then there is such a set of five students (witc)

$\Leftrightarrow$ The number of students who participated in sets of students that drank together a whole glass of beer is five AND there is such a set of five students.

The requirement that the intersection set $\mathcal{A} \cap \mathcal{B}$ is not empty guarantees that the witness condition is automatically satisfied by the $d f i t$ strategy with downward monotone determiners as in (51). This fact is stated formally in the following proposition.

Proposition 2 If a determiner $D$ is downward monotone on its second argument then for all $\mathcal{A}, \mathcal{B} \subseteq \wp(E)$ : if $\operatorname{dfit}(\mathcal{A})(\mathcal{B})$ holds then witc $(D)(\mathcal{A})(\mathcal{B})$ holds.

Thus, the witness condition guarantees "complete collectivity" in cases like (47) but its status as an additional strategy to dfit and the conditioning of the existential operator by non-emptiness of the intersection $\mathcal{A} \cap \mathcal{B}$ avoids problems with nonupward-monotone determiners.

\section{Summary}

By way of summarizing the main proposal of this paper, table 2 gives the options it uses for composing the meanings of a determiner and a noun according to the two relevant features of the noun. In cases I and II, where the noun is in singular, it is translated as an et predicate and the only available option is to compose this predicate directly with the determiner. When the noun is an atom predicate, its singular form's denotation is simply its basic denotation $A$. When the noun is a set predicate in the singular (e.g. colleague), its et denotation is obtained using the $s g$ operator from its basic ett meaning $\mathcal{A}$. Plural nouns in cases III and IV are ambiguous between et predicates and ett predicates. The et reading leads to distributive quantification over atoms. The ett denotation triggers quantification over sets using the $d f i$ strategy, which is responsible for collectivity effects with determiners. An atom predicate gets its ett denotation using the pdist operator as in case III. The dfit strategy leads in this case to a collectivity effect if the NP is 
an argument of a set predicate. If this predicate is an atom predicate, then the process does not generate any collectivity (proposition 1). In case IV the noun is a set predicate, which means that its ett denotation is basic and the et denotation is derived by the $s g$ operator.

\begin{tabular}{|l|l|l|l|l|l|}
\hline & \multicolumn{2}{|l|}{ N Features } & DET & N & NP \\
\hline I & singular & atom & $\bar{D}$ & $A$ & $D(A)$ \\
\hline II & singular & set & $D$ & $s g(\mathcal{A})$ & $D(s g(\mathcal{A}))$ \\
\hline III & plural & atom & $D$ & $A$ & $D(A)$ \\
& & & $D$ & $p \operatorname{dist}(A)$ & $\operatorname{dfit}(D)(p \operatorname{dist}(A))$ \\
\hline IV & plural & set & $\bar{D}$ & $\operatorname{sg}(\mathcal{A})$ & $D(s g(\mathcal{A}))$ \\
& & & $D$ & $\mathcal{A}$ & $\operatorname{dfit}(D)(\mathcal{A})$ \\
\hline
\end{tabular}

Table 2: DET-N meaning composition

\section{Endnotes}

* I thank Gennaro Chierchia, Jaap van der Does, Danny Fox, Ed Keenan, Fred Landman, Remko Scha, Henk Verkuyl and, especially, Johan van Benthem, Christine Brisson, Eyal Hurvitz, Tanya Reinhart, Anna Szabolcsi and Joost Zwarts for remarks and discussions.

1. With the indefinite subjects in (2), some speakers get "generic" readings like to be exactly four students is to be a good team. However, also for such speakers a non-generic reading is still impossible. This can be shown robustly by replacing the predicate by a stage-level predicate like are the team that won the cup yesterday that does not give rise to generic readings. This makes the sentence completely uninterpretable.

2. The condition is not sufficient because: (i) the predicate needs to be a set predicate (cf. (1)). (ii) The resulting proposition should make sense. For instance, a sentence like less than two students are meeting is unacceptable for the same reasons that the sentence John is meeting is unacceptable. This is predicted by the account of section 3 .

3. Note that I assume that the verb lift is a set predicate on its subject argument, and therefore the $s g$ operator is used on the second argument of a basic $e((e t) t)$ denotation to get the $e(e t)$ denotation of this verb in (22b).

4. An open question concerns the strong implication that plural noun phrases as in (i) require the existence of more than one blond students.

(i) The blond students are tall.

An operator like pdist does not exclude singularities from the denotation of the noun and hence (i) is expected to be true in situations where there is only one blond student and this student is tall. Following many works, I assume that the infelicity of (i) in such situations results from pragmatic effects, and not from denotational reasons. 
5. Gennaro Chierchia expressed such an idea in a course given at Utrecht University in winter 1996, relating it to the "copy theory of traces".

6. Dowty's account of all is based on what he calls the subentailments of the predicate. While a predicate like meet is assumed to have distributive subentailments for singular members of its plural argument, predicates like be numerous or be a good team are characterized as "purely collective": they lack distributive subentailments. Dowty does not define this notion and it does not seem that his informal analysis of all can be profitably extended to treat other cases of plural quantification as in (2).

\section{References}

Barker, C. (1992). Group terms in English: representing groups as atoms. Journal of Semantics, 9:69-93.

Bennett, M. (1974). Some Extensions of a Montague Fragment of English. PhD thesis, University of California Los Angeles.

Dowty, D. (1987). Collective predicates, distributive predicates and all. In Proceedings of the Eastern States Conference on Linguistics, ESCOL3.

Fodor, J. D. and Sag, I. (1982). Referential and quantificational indefinites. Linguistics and Philosophy, 5:355-398.

Landman, F. (1989). Groups I \& II. Linguistics and Philosophy, 12:559-605,723744.

Landman, F. (1996). Plurality. In Lappin, S., editor, The Handbook of Contemporary Semantic Theory. Blackwell.

Link, G. (1983). The logical analysis of plurals and mass terms: a lattice theoretical approach. In Bauerle, R., Schwarze, C., and von Stechow, A., editors, Meaning, Use and Interpretation of Language. De Gruyter, Berlin.

Reinhart, T. (1997). Quantifier scope: how labor is divided between QR and choice functions. Linguistics and Philosophy, 20:335-397.

Scha, R. (1981). Distributive, collective and cumulative quantification. In Groenendijk, J., Stokhof, M., and Janssen, T. M. V., editors, Formal Methods in the Study of Language. Mathematisch Centrum, Amsterdam.

Schwarzschild, R. (1996). Pluralities. Kluwer, Dordrecht.

Szabolcsi, A. (1997). Strategies for scope taking. In Szabolcsi, A., editor, Ways of Scope Taking. Kluwer, Dordrecht.

van der Does, J. (1993). Sums and quantifiers. Linguistics and Philosophy, 16:509_ 550.

Winter, Y. (1997). Choice functions and the scopal semantics of indefinites. Linguistics and Philosophy, 20:399-467.

Utrecht Institute of Linguistics OTS

Trans 10

3512 JK Utrecht

The Netherlands

E-mail: yoad.winter@let.uu.nl

WWW: http://www-uilots.let.uu.nl/ ${ }^{\sim}$ Yoad.Winter/ 